\title{
Therapeutic Antisense Targeting of Huntingtin
}

\author{
Anne V. Smith ${ }^{1}$ and Sarah J. Tabrizi ${ }^{2}$
}

Antisense oligonucleotides (ASOs) are a relatively new therapeutic entity that utilizes short chemically modified strands of DNA in targeted interactions with RNA to modulate the type or amount of resultant protein. This brief review summarizes the preclinical, translational, and early clinical development of an ASO designed to reduce the production of the disease-causing protein in Huntington's disease, an inherited neurodegenerative disease.

Keywords: antisense oligonucleotide, clinical trial, Huntington's disease, huntingtin lowering

\section{Introduction}

$\mathbf{H}$ UNTINGTON's DISEASE (HD) IS an autosomal dominant neurodegenerative disorder. Approximately 30,000 people in the United States have HD, and many more are at risk of developing HD. Disease onset usually occurs in midadult life and follows a 15- to 20-year inexorable course to death. A less common form-juvenile HD-begins in childhood and progresses more rapidly, typically resulting in death $\sim 10$ years from motor symptom onset. Current treatments for HD are limited to symptomatic therapies, as no treatment has been shown to prevent onset or to slow progression (Bates et al., 2015).

HD is often depicted as a combination of Parkinson's disease, Alzheimer's disease (AD), and amyotrophic lateral sclerosis (ALS) because it manifests in physical changes (abrupt involuntary movements, coordination deficits, speaking difficulties, and swallowing difficulties), cognitive decline (deterioration in communication, planning, decision-making, and memory), and behavioral changes (irritability, aggression, mood swings, and apathy). Similar to Parkinson's disease, $\mathrm{AD}$, and ALS, the pathology of HD is characterized by aberrant aggregation of protein in brain cells. Unlike these other diseases, every case of HD is caused by a single genetic mutation-a CAG repeat expansion in HTT, the gene that encodes the huntingtin protein. The abnormal gene results in production of mutant huntingtin protein (mHTT) containing an expanded polyglutamine tract, which leads to neuronal dysfunction and death through toxic gain-offunction mechanisms.

Traditional drug discovery is a resource intensive effort with pharmacologists, cell and molecular biologists, me- dicinal chemists, pharmacokineticists and toxicologists all serving important roles. With the use of high-throughput screening techniques, thousands of small molecules can be evaluated for activity toward specific biological targets to identify leads worthy of further testing (Dandapani et al., 2012). However, these methods are low yield, and targets are generally limited to select classes of proteins with known structures and well-defined binding sites. Fortunately, advances in genomics have accelerated our ability to link genetic targets to human disease and development of new therapeutic platforms are expanding the number of druggable targets, creating an environment conducive to intelligent design of highly specific potentially diseasemodifying drugs.

RNA-targeted therapeutics, such as antisense oligonucleotides (ASOs), epitomize informed drug design. Over the past two decades, ASO technology matured through foundational research on ASO mechanisms, pharmacokinetics (PKs), and safety; and the technology attained viability through medicinal chemistry inventions imparting desirable drug-like properties (Crooke et al., 2018). Recent new drug approvals -including nusinersen, an ASO for the treatment of spinal muscular atrophy-demonstrate the potential of ASOs to effect disease modification in a neurological disease.

Given the monogenic nature of HD, where disease pathology is primarily due to production of mHTT, inhibition of HTT expression represents a promising therapeutic strategy. ASO technology provides a direct route to inhibition of HTT expression by targeting HTT RNA for destruction, thus preventing translation of mHTT and targeting the primary disease mechanism.

\footnotetext{
${ }^{1}$ Ionis Pharmaceuticals, Carlsbad, California.

${ }^{2}$ Huntington's Disease Centre, Department of Neurodegenerative Disease, University College London (UCL) Queen Square Institute of Neurology, and the UK Dementia Research Institute at UCL, London, United Kingdom.
} 


\section{Antisense Oligonucleotides}

ASOs are short single-stranded oligomers comprising chemically modified nucleotides that bind to RNA to modulate its function. The order of nucleotides can be designed to enable high specificity to a target RNA, and selection and location of chemical modifications along the ASO dictate postbinding functional modulations. Broadly, ASO mechanisms can be categorized as either promoting the target RNA's degradation through endogenous enzymes, such as RNase H1, or interfering with the target RNA's function without promoting degradation, such as translation arrest or modulation of RNA processing (Bennett et al., 2017). Figure 1 depicts the ASO RNase H1 mechanism, which is utilized in this program to degrade HTT RNA.

\section{Foundation for Clinical Testing in HD}

Evidence supporting pharmacological lowering of HTT as a promising therapeutic strategy for HD has accumulated for the past decade, with data emerging from multiple laboratories using various methods to lower HTT in animal models of HD (Keiser et al., 2016). In a series of preclinical experiments, ASOs were shown to effect long-lasting reduction of HTT RNA and protein throughout the central nervous system; effects were dose-dependent with a maximal achievable reduction of $\sim 75 \%$, although even modest reductions of $\sim 35 \%$ were associated with phenotypic and survival benefits (Kordasiewicz et al., 2012; Stanek et al., 2013; Southwell et al., 2018).

Before testing an HTT-lowering ASO in humans, extensive toxicology, PK, and pharmacodynamic (PD) preclinical testing of the human candidate ASO was undertaken. ASO potency and tolerability can vary widely depending on nucleotide sequence and chemical modifications, so a broad screening of ASOs-all homologous to human HTT - was conducted to select a suitable human candidate. Additional animal testing was conducted to allow for construction of a preclinical PK/PD model relating ASO dose level to changes in HTT in brain tissue and in cerebrospinal fluid (CSF). This model was used to inform selection of the ASO doses to be used in the clinic and to allow for extrapolation from PD effects that are measurable in the clinic (such as change in CSF HTT) to PD effects of interest (such as change in HTT RNA and HTT protein in brain tissue).

In parallel with preclinical testing of the human candidate ASO, a clinical development plan was created. Although large long clinical trials will ultimately be necessary to determine if the candidate ASO is efficacious and safe, valuable insights into the ASO's promise-and to the overriding concept of HTT lowering as a therapeutic strategy for HD — can be assessed in a much smaller setting. Accordingly, a short first-in-human safety trial was designed

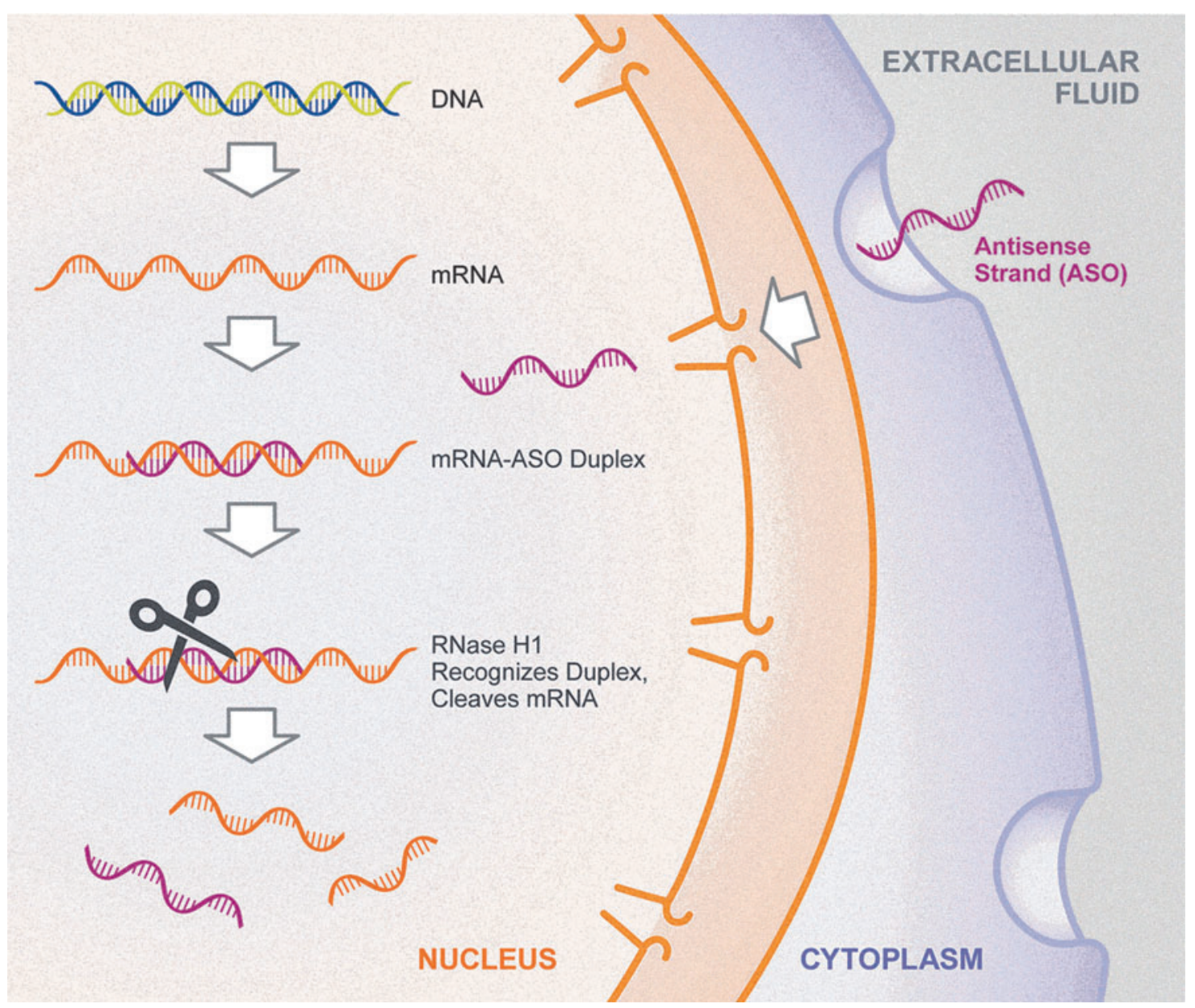

FIG. 1. RNase H1 ASO mechanism of action. ASO, antisense oligonucleotide. 
A Concentration of Mutant HTT in CSF of Individual Patients over Time, According to Dose Group

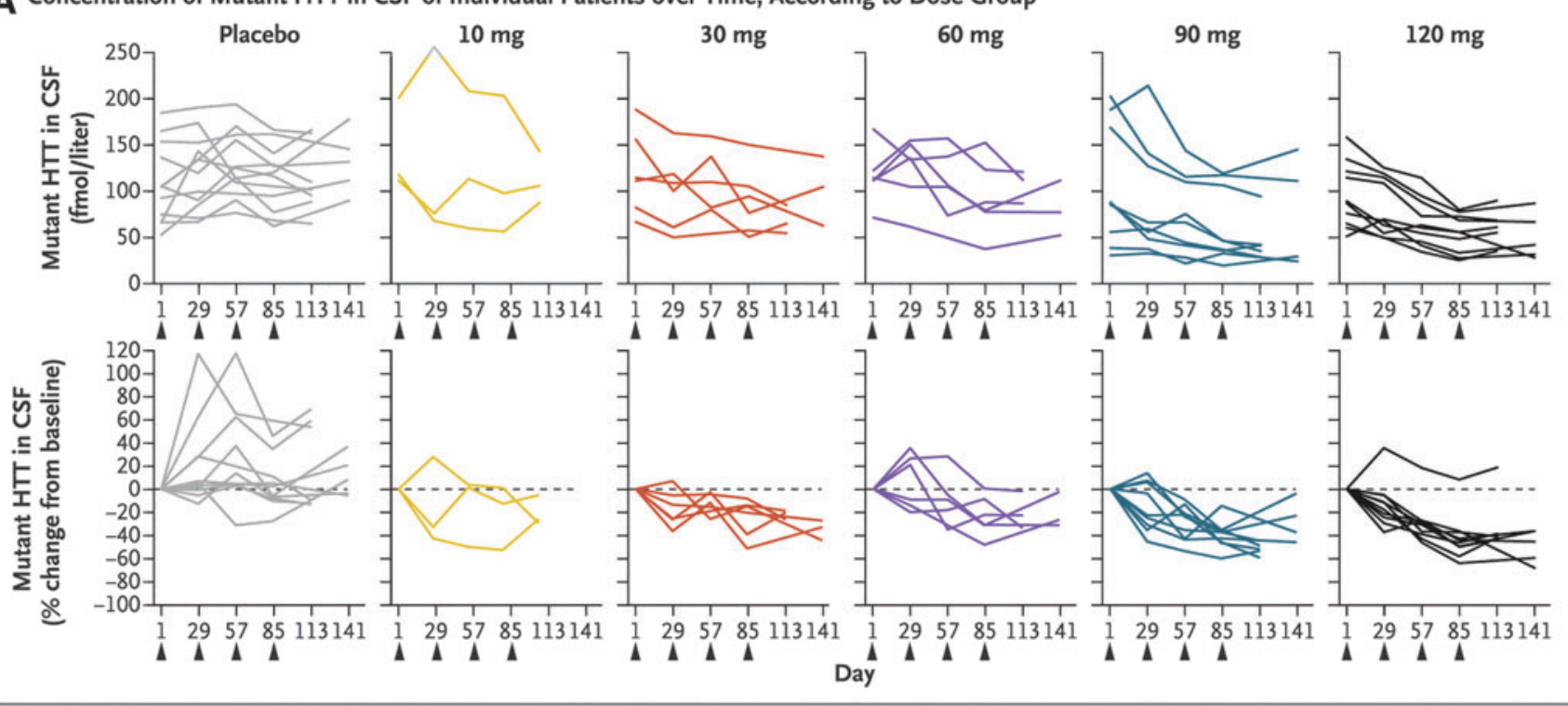

B Percentage Change in CSF Concentration of Mutant HTT, According to Dose Group

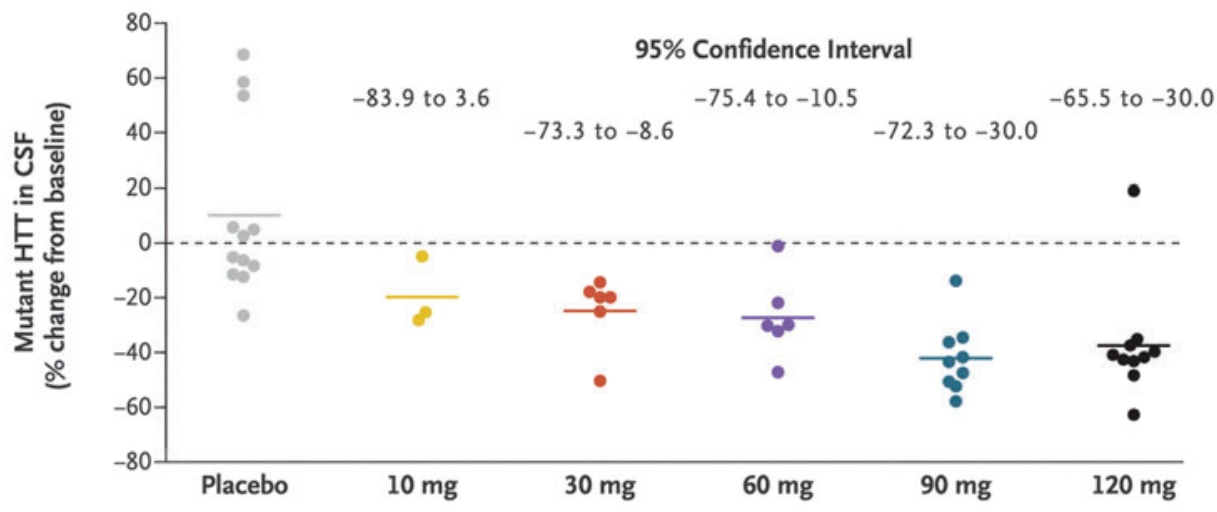

C Mean CSF Concentration of Mutant HTT and Percentage Change from Baseline, According to Dose Group
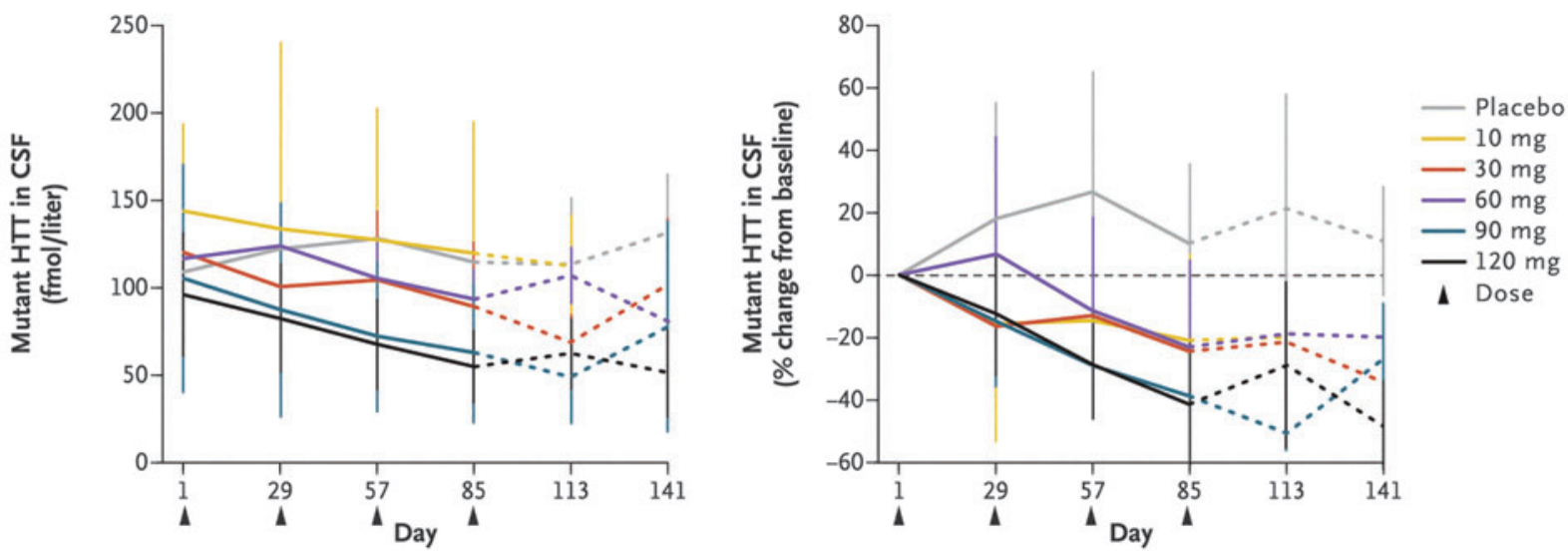

FIG. 2. Effect of $\mathrm{HTT}_{\mathrm{Rx}}$ on concentrations of mHTT in CSF. (A) Concentrations of mHTT in CSF over time for individual patients in each dose group; absolute values are shown in the top graphs, and the percentage changes from baseline (dotted line) are shown in the bottom graphs. Arrowheads indicate the 4 days on which study drug was administered. (B) Percentage change in the concentration of mHTT in CSF from baseline (dotted line) to the last available time point 28 days after the previous dose (i.e., either day 113 for the patients who underwent CSF sampling at day 113 or day 85 for the other patients). Circles indicate individual patients, and horizontal lines indicate group means; $95 \%$ confidence intervals are also shown for the active-agent dose groups. (C) Mean concentration of mHTT in CSF (left) and the mean percentage change from baseline (right) over time according to dose group. Error bars indicate the standard deviation. Samples from days 113 and 141 were each obtained in a randomized subgroup of patients (dotted lines). Figure from Tabrizi et al. (2019). Reprinted with permission from Massachusetts Medical Society. CSF, cerebrospinal fluid; mHTT, mutant huntingtin protein. 
to evaluate the tolerability of repeated intrathecal injections of the ASO and to obtain exploratory measures of ASO pharmacology.

The design of the first clinical trial was a randomized double-blinded placebo-controlled multiple ascending dosedose multicenter phase $1 / 2$ a study conducted in $46 \mathrm{HD}$ patients with early-stage disease at nine international centers (NCT02519036). Study participants were assigned to ASO $\left(\mathrm{HTT}_{\mathrm{Rx}}\right.$, also known as ISIS 443139 or RG6042) or placebo (artificial CSF) at a ratio of 3:1 within each of five dosing cohorts $(10,30,60,90$, or $120 \mathrm{mg})$. Each participant received four lumbar intrathecal bolus injections of $\mathrm{HTT}_{\mathrm{Rx}}$ or placebo at 4-week intervals followed by a 4-month untreated followup period. A CSF sample was collected before each study drug injection, and an additional CSF sample was collected either 4 or 8 weeks after the last dose of study drug. The primary objective was evaluation of the safety and tolerability of $\mathrm{HTT}_{\mathrm{Rx}}$. Other objectives included characterization of CSF $\mathrm{PKs}$ of $\mathrm{HTT}_{\mathrm{Rx}}$ and exploration of the effects of $\mathrm{HTT}_{\mathrm{Rx}}$ on PD biomarkers and clinical endpoints relevant in HD, including mHTT concentration in CSF and functional assessments commonly used in HD.

\section{Key Results of the Clinical Trial}

The trial and all results are described in detail in Tabrizi et al. (2019). Overall, $\mathrm{HTT}_{\mathrm{Rx}}$ was well tolerated. All patients received all scheduled doses of assigned treatment, and all patients completed the trial according to the protocol. Adverse events were generally mild and unrelated to study drug. The only serious adverse event was admission of a patient in the placebo group for a transient postural headache determined to be unrelated to study drug. There were no clinically relevant adverse laboratory parameter changes and no evidence of renal toxicity, hepatotoxicity, or thrombocytopenia during the study.

$\mathrm{HTT}_{\mathrm{Rx}}$ treatment resulted in significant dose-dependent reduction in CSF mHTT. At the two highest doses, 90 and $120 \mathrm{mg} / \mathrm{month} \mathrm{HTT}_{\mathrm{Rx}}$, a mean reduction in CSF mHTT of $40 \%$ was observed, with a maximum individual reduction of $>60 \%$ (Fig. 2A, B) and a downward trajectory suggesting steady-state maximal reduction of CSF mHTT was not reached during this short study (Fig. 2A, C).

$\mathrm{HTT}_{\mathrm{Rx}}$ concentrations were well aligned with predictions from the preclinical PK/PD model. Using the model to estimate ASO effects in brain tissue, $40 \%$ reduction in CSF mHTT is expected to reflect 55-70\% lowering of mHTT in cortical tissue and 20-35\% lowering of mHTT in striatal tissue; and $60 \%$ lowering in CSF mHTT is expected to reflect $70-85 \%$ lowering of mHTT in cortical tissue and 35$50 \%$ lowering of mHTT in striatal tissue. Sustained mHTT reduction of this magnitude in tissue reaches or exceeds the threshold shown to produce significant improvements in motor function and survival in transgenic mouse models of HD (Kordasiewicz et al., 2012; Keiser et al., 2016). Thus, the central nervous system (CNS) mHTT lowering achieved in the high-dose groups in this study is believed to be pharmacologically relevant.

Functional, cognitive, psychiatric, and neurological clinical outcomes were generally unchanged during the study, and no differences were observed between placebo-treated patients and any $\mathrm{HTT}_{\mathrm{Rx}}$ dose group. This short study was not designed or sufficiently powered to allow for evaluation of the effect of $\mathrm{HTT}_{\mathrm{Rx}}$ on disease biomarkers or clinical measures of HD. HD is a slow-progressing disease, with changes on standard outcomes generally occurring over years, not weeks.

\section{Conclusions}

Since the discovery of the HTT gene in 1993 , methods to reduce its toxic product, $\mathrm{mHTT}$, have been sought. The clinical study described was the first to demonstrate pharmacologically induced reduction of mHTT in CSF, which likely reflects a reduction of $\mathrm{mHTT}$ in CNS tissue. Reaching this milestone is the result of a comprehensive drug discovery and preclinical program to identify an ASO that safely, potently, and specifically suppresses HTT production, thus demonstrating that robust early development efforts can facilitate successful translation to the clinic. Larger longer-term studies are underway to determine whether $\mathrm{HTT}_{\mathrm{Rx}}$-mediated CNS mHTT reduction effects meaningful changes to disease course (NCT03342053, NCT03761849; sponsored by Hoffman-La Roche).

The observations in this study may have important ramifications well beyond the HD field. This study was not just the first to demonstrate ASO-mediated protein suppression in the CNS of patients with HD, but the first to demonstrate ASO-mediated protein suppression in individuals with any neurodegenerative disease. Recently, similarly promising results have been reported for an SOD1-targeting ASO in ALS patients (Miller et al., 2019). HD is often depicted as a combination of Parkinson's disease, AD, and ALS — a stark reminder of the current lack of disease-modifying treatments for any of these conditions. In the coming years, we look to ASOs as a promising therapeutic modality to meet this need not only for HD but also for Parkinson's disease, AD, ALS, and other neurodegenerative diseases associated with aberrant protein production or accumulation.

\section{Acknowledgments}

The authors thank the patients and their companions who participated in the study and the principal investigators and study teams for executing the study. The authors also thank Wanda Sullivan for creating Figure 1 and Frank Bennett and Roger Lane for critical review of this manuscript.

\section{Disclosure Statement}

S.J.T. is partly supported by the UK Dementia Research Institute that receives its funding from DRI Ltd., funded by the UK Medical Research Council, Alzheimer's Society, and Alzheimer's Research UK; and research grant funding from the Wellcome Trust (ref. 200181/Z/15/Z). In the past 3 years, through the offices of UCL Consultants Ltd., a wholly owned subsidiary of University College London, S.J.T. has undertaken consultancy services for Alnylam Pharmaceuticals, Inc., Annexon, Inc., DDF Discovery Ltd., F. HoffmannLa Roche Ltd., Genentech, GSK, Heptares Therapeutics Ltd., Ixico, Novartis Pharma, Shire Human Genetic Therapies, Inc., Takeda Pharmaceuticals Ltd., Teva Pharmaceuticals Ltd., Triplet Therapeutics, UCB Pharma S.A., University College Irvine, and Vertex Pharmaceuticals, Inc. S.J.T. has also 
provided consultancy services directly to Alphasights, Decision Resources Group, and Triangle Insights Group.

A.V.S. is a salaried employee of Ionis Pharmaceuticals.

\section{Funding Information}

The study was funded by Ionis Pharmaceuticals and F. Hoffmann-La Roche Ltd.

\section{References}

Bates, G.P., Dorsey, R., Gusella, J.F., Hayden, M.R., Kay, C., Leavitt, B.R., et al. (2015). Huntington disease. Nat Rev Dis Primers 1, 15005.

Bennett, C.F., Baker, B.F., Pham, N., Swayze, E., and Geary, R.S. (2017). Pharmacology of antisense drugs. Annu Rev Pharmacol Toxicol 57, 81-105.

Crooke, S.T., Witztum, J.L., Bennett, C.F., and Baker, B.F. (2018). RNA-targeted therapeutics. Cell Metab 27, 714-739.

Dandapani, S., Rosse, G., Southall, N., Salvino, J.M., and Thomas, C.J. (2012). Selecting, acquiring, and using small molecule libraries for high-throughput screening. Curr Protoc Chem Biol 4, 177-191.

Keiser, M.S., Kordasiewicz, H.B., and McBride, J.L. (2016). Gene suppression strategies for dominantly inherited neurodegenerative diseases: lessons from Huntington's disease and spinocerebellar ataxia. Hum Mol Genet 25(R1), R53-R64.

Kordasiewicz, H.B., Stanek, L.M., Wancewicz, E.V., Mazur, C., McAlonis, M.M., Pytel, K.A., et al. (2012). Sustained therapeutic reversal of Huntington's disease by transient repression of huntingtin synthesis. Neuron 74, 1031-1044.

Miller, T., Cudkowicz, M., Shaw, P.J., Graham, D., Fraddette, S., Houshyar, H., et al. (2019). Safety, PK, PD, and exploratory efficacy in single and multiple dose study of a SOD1 antisense oligonucleotide (BIIB067) administered to participants with ALS. Presented at: 2019 American Academy of Neurology Annual Meeting, May 4-10, 2019; Philadelphia, PA (Abstract).
Southwell, A.L., Kordasiewicz, H.B., Langbehn, D., Skotte, N.H., Parsons, M.P., Villanueva, E.B., et al. (2018). Huntingtin suppression restores cognitive function in a mouse model of Huntington's disease. Sci Transl Med 10, pii: eaar3959.

Stanek, L.M., Yang, W., Angus, S., Sardi, P.S., Hayden, M.R., Hung, G.H., et al. (2013). Antisense oligonucleotide-mediated correction of transcriptional dysregulation is correlated with behavioral benefits in the YAC128 mouse model of Huntington's disease. J Huntingtons Dis 2, 217-228.

Tabrizi, S.J., Leavitt, B.R., Landwehrmeyer, G.B., Wild, E.J., Saft, C., Barker, R.A., et al. (2019). Targeting huntingtin expression in patients with Huntington's disease. N Engl J Med 380, 2307-2316.

Address correspondence to: Anne V. Smith, PhD Ionis Pharmaceuticals Carlsbad, CA 92010

E-mail: asmith@ionisph.com

Sarah J. Tabrizi, FRCP, PhD, FMedSci

Huntington's Disease Centre Department of Neurodegenerative Disease UCL Queen Square Institute of Neurology Box 104, National Hospital for Neurology and Neurosurgery Queen Square London WC1N 3BG United Kingdom

E-mail: s.tabrizi@ucl.ac.uk

Received for publication October 31, 2019; accepted October 31, 2019. 